\title{
NOTAS
}

\section{HERNÁN CORTÉS EN SUS CARTAS DE RELACIÓN: LA CONFIGURACIÓN LITERARIA DEL HÉROE}

La figura histórica del conquistador Hernán Cortés es sin duda una de las más controvertidas en el ámbito latinoamericano. Basta recordar los versos de Pablo Neruda en el Canto general ("Cortés no tiene pueblo, es rayo frío, / corazón muerto en la armadura") para reconocer lo que Octavio Paz definía como el "mito negro" de Cortés, un mito que desde la Independencia se ha vinculado a la barbarie de la conquista española. Sin embargo, es incluso en ese período formativo de las nuevas naciones, tan convulso ideológica y políticamente, y en el propio ámbito mexicano, donde podemos descubrir todavía el respeto por el hombre que fue considerado por sus contemporáneos un nuevo Julio César. La casi paradójica exclamación del liberal Manuel Orozco y Berra a mediados del siglo xix, "Nuestra admiración para el héroe, nunca nuestro cariño para el conquistador" , expresaba entonces el juego de contradicciones que la heroicidad admirable de este "negro" personaje ha generado en la cultura mexicana hasta nuestros días, y que Paz sintetiza asimismo de manera rotunda: "Fue un hombre extraordinario, un héroe en el antiguo sentido de la palabra. No es fácil amarlo, pero es imposible no admirarlo" 2 .

El presente trabajo se centra en esa visión heroica del conquistador que subyace a las dos frases citadas, y más concretamente al texto que la generó: las Cartas de relación de Hernán Cortés ${ }^{3}$, en las que el autor configuró una imagen idealizada de sí mismo que fue asumida a su vez por la literatura (y por buena parte de la historiografía) colonial posterior, y de la que no han podido sustraerse ni sus más modernos biógrafos. Más allá de su valor histórico (pero en estrecha

${ }^{1}$ Cit. en José Luis Martínez, Hernán Cortés, F.C.E., México, 1993, p. 833.

2 Octavio Paz, "Hernán Cortés: exorcismo y liberación" (El País, 12 de octubre de 1985), en El peregrino en su patria, F.C.E., México, 1988, p. 103.

${ }^{3}$ Recordemos, en cualquier caso, que las Cartas constituyen sólo una parte (aunque muy significativa) del corpus textual cortesiano (cf. Hernán Cortés, Documentos cortesianos, ed. J.L. Martínez, UNAM-F.C.E., México, 1991-92). 
relación con éste), las Cartas de relación se nos presentan como un texto fundador de la literatura colonial en la medida en que Cortés logra en ellas la creación de un modelo heroico de conquistador que, por un lado, se construye a su vez a partir de modelos previos de la tradición cultural europea y, por otro, delimita ya las distintas vertientes del héroe de su tiempo, simbolizando en su figura todo un período de transición entre el mundo medieval y el renacentista. En las siguientes páginas, intentaré apuntar algunas ideas sobre la manera en que convergen en los textos cortesianos los dos aspectos señalados, esto es, los distintos modelos de los que parte el autor y los rasgos seleccionados por éste en una construcción literaria de sí mismo que resultará paradigmática para la valoración posterior de un personaje histórico y mítico.

\section{EL INGRESO EN LA HISTORIA Y LA BÚSQUEDA DE MODELOS}

Como sabemos, a lo largo de las cinco Cartas de relación ${ }^{4}$, Cortés narró los acontecimientos que marcaron el ascenso y declive de su singular figura en apenas siete años: los inicios de la expedición que, bajo su mando, salió de Cuba en 1519 hacia el actual territorio mexicano y culminó con la fundación de la Veracruz (asunto de la Primera relación, hoy perdida y sustituida por la llamada Carta de Veracruz ${ }^{5}$ ); la marcha sobre México-Tenochtitlán, el encuentro con Moctezuma, la prisión del monarca y la rebelión de los mexicas en su ausencia que provocaría la llamada "Noche triste" (acontecimientos descritos en la Segunda relación); el asedio militar a la capital, su destrucción y el asentamiento del poder español en México (objeto de la Tercera relación); las conquistas posteriores y sus primeros trabajos de organización administrativa como gobernador de la Nueva España (que son el tema de la Cuarta relación); y la fracasada expedición a Honduras, seguida del juicio de residencia que se abrió contra él en 1526 (hechos ambos que se describen en la Quinta relación).

\footnotetext{
${ }^{4}$ Hernán Cortés, Cartas de relación, ed. A. Delgado Gómez, Castalia, Madrid, 1993 (en adelante, el texto se citará por esta edición).

${ }^{5}$ La existencia de la Primera relación queda atestiguada por Cortés, por López de Gómara (quien es probable que incluso la leyera antes de escribir su Conquista de México) y por Bernal Díaz del Castillo (aunque éste reconoce no haberla visto); sin embargo, la postura de los investigadores sobre si Cortés escribió o no esta relación no es unívoca, como tampoco lo es la referente a la posibilidad de analizar la Carta de Veracruz como texto cortesiano. Cf., a este respecto, entre otros trabajos, Julio Caillet-Bois, "La primera carta de relación de Hernán Cortés", Revista de Filología Hispánica, 3 (1941), 50-54; José Toribio Medina, Ensayo bio-bibliográfico sobre Hernán Cortés, introd. G. Feliu Cruz, Fondo histórico y bibliográfico José Toribio Medina [Ed. Nascimento], Santiago de Chile, 1952, p. 2; José Luis Martínez, op. cit., pp. 197-201, y Ángel Delgado, introd. a Cartas, pp. 37-41.
} 
Una lectura siquiera superficial de estos textos permite deducir que, al menos a partir de la Segunda relación, Cortés fue consciente de estar protagonizando unos hechos que entrarían a formar parte de la Historia y, por ello, en especial en ésta y en la siguiente carta (pero también, de diferente forma, en el conjunto de su obra $^{6}$ ) intentó no sólo definir su proyecto de conquista como una gran hazaña del Imperio español sino también presentarse a sí mismo como el gran héroe protagonista de dicho proyecto, un héroe para la Fama.

En su libro, Discurso narrativo de la conquista de América ${ }^{7}$, Beatriz Pastor analiza esta imagen heroica de Cortés en las Cartas a partir de lo que la investigadora española define como un proceso de ficcionalización del discurso narrativo que permite a su autor transformar la rebelión en servicio (recordemos que Cortés parte a la conquista de las nuevas tierras cuando Diego Velázquez ha revocado su nombramiento y, por tanto, desobedeciendo el poder real) y mostrarse no como rebelde sino como modelo, como héroe capaz de enfrentarse al infame Velázquez y conquistar un gran imperio para su rey. Pastor muestra de qué manera Cortés va creando una imagen de sí mismo como héroe que se sitúa en el punto de convergencia entre la concepción medieval del vasallo y la renacentista del perfecto gobernante y que se configura, desde su perspectiva, en dos fases: en primer lugar, Cortés realiza (en las tres primeras cartas) un proceso de mitificación de la propia figura que culmina con la caída de Tenochtitlán y que corresponde en sus rasgos esenciales al modelo renacentista reflejado en El Príncipe de Maquiavelo, ya que se describe a sí mismo como perfecto hombre de guerra, político y gobernante; en segundo lugar, y dado que esa imagen idealizada podía convertirlo en un hombre peligroso para la Corona, Cortés problematiza y humaniza paulatinamente su figura a lo largo de las cinco cartas, integrando este modelo de héroe renacentista en una estructura medieval de vasallaje y providencialismo. De este modo, según Pastor,

Al elegir narrar dentro del marco documental de la relación una versión personal y mitificadora de la conquista de México, Cortés rompe los límites del discurso de la "historia de verdad" y los vacía de contenido, convirtiendo sus formas en una convención literaria que es parte

${ }^{6}$ A pesar de haber sido escritas en etapas distintas de la vida del autor y con diversas motivaciones (e incluso de la sustitución de la Primera relación por la llamada Carta de Veracruz), las cinco cartas, tal como hoy se conocen, mantienen, como ha señalado entre otros Ángel Delgado, una unidad temática, ideológica y estilística (véase Delgado en ibid., p. 37).

${ }^{7}$ Beatriz Pastor, "Hernán Cortés: la ficcionalización de la Conquista y la creación del modelo del conquistador", Discurso narrativo de la conquista de América, Casa de las Américas, Santiago, 1983, pp. 113-233. 
integrante de la estructura narrativa del discurso fundamentalmente ficcional que introducen y configuran ${ }^{8}$.

Si bien la conclusión de Pastor sobre el carácter ficcional de las Cartas de relación puede ser discutible, lo que me parece más valioso de su argumentación es la manera en que llama la atención sobre dos elementos propiamente literarios del texto: por un lado, esa fuerte presencia del yo en su escritura, esa dimensión autobiográfica que, como explicará posteriormente Liria Evangelista, se incorpora a la narración histórica modificándola necesariamente, convirtiéndola en "relato de una vida"; por otro lado, la utilización por parte de Cortés de modelos histórico-literarios propios de la tradición europea al presentarse como personaje en su obra ${ }^{10}$. Me parece, por ello, útil matizar y ampliar los planteamientos citados para determinar los que considero serían los modelos de la escritura cortesiana (presentes en algún caso a partir de la posible lectura de un texto, pero, sobre todo, como parte del horizonte cultural del autor) y los rasgos de esa imagen heroica del yo construida en el texto (en buena medida como deuda a dichos modelos).

Por lo que respecta a los modelos histórico-literarios que se entrecruzan y se complementan en las Cartas de relación, creo posible distinguir tres líneas básicas de confluencia:

La primera de dichas líneas es el ideal político y militar de la época, llevado hasta sus últimas consecuencias en un texto contemporáneo a las Cartas como es El Príncipe de Maquiavelo. Si bien Cortés no pudo haber leído la obra del canciller italiano (que, aunque escrita en 1513, se publicó en 1532), el análisis de las Cartas de relación a partir de los presupuestos maquiavélicos puede arrojar mucha luz sobre la imagen que el conquistador pretende dar de sí mismo en la medida en que, como ha señalado Beatriz Pastor,

Esa capacidad de análisis concreto de una realidad concreta sobre la que se levanta la formulación de la filosofía del racionalismo político del renacimiento es la misma que está en la base de los términos específicos de la caracterización de Cortés como modelo ${ }^{11}$.

${ }^{8}$ Ibid., p. 152.

${ }^{9}$ Véase Liria Evangelista, "Hernán Cortés: los viajes del yo”, A, julio-diciembre de 1999, núm. 480 p. 35.

${ }^{10}$ En este sentido, es de interés el trabajo de Elena Pellús, Hernán Cortés desde la historiografía de Indias: estudio y traducción de "De rebus gestis Fernandini Cortesii”, Universidad, Alicante, 2002 (memoria de licenciatura inédita); véanse en especial las pp. 39-71, donde la imagen de Cortés como figura emblemática de su época se analiza a partir de la confrontación con textos como El Príncipe o Las siete partidas.

${ }^{11}$ Pastor, op. cit., p. 191 (véase su análisis en pp. 190-220). 
El texto de Maquiavelo, verdadero manual sobre la conquista de nuevos territorios y el asentamiento del poder en éstos, admite -como han planteado ya diversos autores- numerosas confrontaciones con la actuación de Cortés tanto durante la marcha sobre México-Tenochtitlán y el asedio a la ciudad como en su posterior gobierno de la Nueva España ${ }^{12}$.

El segundo modelo es el mundo clásico, recuperado por la cultura renacentista (y específicamente por Maquiavelo ${ }^{13}$ ), en el que se inscriben los grandes héroes míticos e históricos del pasado (Aquiles, Eneas, Alejandro Magno...) y que aporta el modelo textual más significativo del gran conquistador que decide convertirse en historiador de sí mismo: los Comentarios a la guerra de las Galias de Julio César.

César fue el héroe con quien más compararon a Cortés los cronistas y poetas de los siglos posteriores ${ }^{14}$. Conocemos el interés del conquistador por la historia romana, sobre la cual debió leer al menos a Salustio y/o a Plutarco ${ }^{15}$, y, aunque no es posible afirmar que Cortés leyera el texto de César (que sí sabemos era una de las lecturas de cabecera de Carlos $\mathrm{V}^{16}$ ), hay una evidente afinidad temática y estilística entre su obra y los Comentarios a la guerra de las Galias, ya señalada en su momento por autores como Menéndez Pelayo o Zamora

${ }^{12}$ Así, por ejemplo, José Valero Silva, en el capítulo de su libro, El legalismo de Hernán Cortés como instrumento de su conquista (UNAM, México, 1965), dedicado a la visión política y militar de Cortés, lo confronta con El Príncipe (véanse pp. 37-50), y Marco A. Almazán, en su artículo "Hernán Cortés: Virtu vs. Fortuna" (Journal of American Culture, 1997, núm. 20, pp. 131-137), retoma algunos conceptos maquiavélicos para intentar demostrar cómo, a pesar de su inteligencia y valentía, Cortés cometió una serie de errores políticos que hicieron esas cualidades insuficientes para controlar los efectos de la fortuna.

${ }^{13}$ Recordemos que éste recomienda a los príncipes "leer historia y de ella tomar en consideración las acciones de los hombres sobresalientes... y tener siempre presentes sus hazañas y modos de actuar; como se dice de Alejandro, que imitaba a Aquiles; César a Alejandro; Escipión a Ciro" (Nicolás Maquiavelo, El Príncipe, Istmo, Madrid, 2000, p. 118).

${ }^{14}$ Véase Winston Reynolds, "Hernán Cortés y los héroes de la Antigüedad", Revista de Filología Hispánica, 45 (1962), pp. 259-271, reeditado con ligeras variantes en Hernán Cortés en la literatura del Siglo de Oro, Editora Nacional, Madrid, 1978, pp. 115-126.

${ }^{15}$ Sólo así se explicaría, como ha señalado José Luis Martínez, la referencia de Bernal a la cita que Cortés hace de memoria de una anécdota sobre Mario y Sila que aparece en La guerra de Jugurta y en las Vidas paralelas (en concreto en el libro dedicado a Sila); cf. Martínez, op. cit., pp. 850-851. Por lo que respecta tanto a la referencia anterior como en concreto a la biografía de César, es un dato de interés que las Vidas paralelas de Plutarco estuvieran publicadas en Sevilla al menos desde 1491 (en la traducción de Alfonso de Palencia, Cuatro Compañeros Alemanes, Sevilla; véase Francisco VINDEL, El arte tipográfico en España durante el siglo XV. Sevilla y Granada, Padilla, Sevilla, 1989).

${ }^{16}$ Como ha explicado Manuel Fernández Álvarez, los Comentarios fue "uno de los escasos libros que le acompañaron hasta el último retiro de Yuste" (Carlos V. El César y el hombre, Espasa Calpe, Madrid, 2000, p. 180). 
Vicente $^{17}$. No debemos olvidar, en cualquier caso, que la lectura de Salustio pudo ofrecer a Cortés otros modelos de actuación, sobre todo en el caso de La guerra de Jugurta, guerra de alianzas y traiciones, de rencillas entre líderes de un mismo bando, en la que un hombre hábil y astuto logra enfrentarse (a pesar del fracaso final) a todo un imperio.

El tercer modelo, situado en el contexto específico de la cultura castellana, es el de la gran gesta nacional de la Reconquista y, con ella, la figura central del Cid, el vasallo ejemplar que dedica su vida a ganar nuevas tierras para su rey, cuya imagen heroica fue trasmitida tanto a través de las crónicas (alfonsíes y post-alfonsíes) como del Romancero $^{18}$. Las cualidades morales, políticas y militares del héroe castellano, unidas a la inquebrantable lealtad a su rey, debieron ser sin duda un referente importante para Cortés -como ahora veremos-, a pesar de que los autores posteriores prefirieran, de manera general, equipararlo con personajes del mundo clásico.

\section{LA CONSTRUCGIÓN DE LA IMAGEN HEROICA}

Que los modelos citados determinaron la construcción autobiográfica del héroe por parte de nuestro autor lo demuestran los rasgos propios de dicha construcción, rasgos que definen a un personaje que es a un tiempo perfecto militar, gobernante, vasallo y cristiano:

${ }^{17}$ Menéndez Pelayo destacó la manera en que la escritura "nerviosa y viril, rápida y sobria" de las Memorias de César se había reproducido, "a través de los siglos, sin necesidad de imitación directa... en las Cartas de Relación de Hernán Cortés"; para Zamora Vicente, las Cartas "atestiguan [a Cortés] como conocedor del estilo de César, al que habría conocido en sus estudios de Salamanca" (cit. en Hans Flasche, "Problemas de estructura sintáctica que presentan las cartas de Hernán Cortés, dirigidas a Carlos V", Románica [La Plata, Argentina], 3, 1970, pp. 148-149). Sobre las relaciones entre ambos autores y sus textos pueden consultarse, además, los trabajos de Manuel Alcalá: César y Cortés, Jus, México, 1950; "Los Comentarios de Julio César y las Cartas de relación de Hernán Cortés”, Acta Salmanticensia. Filosofía y Letras, 10 (1956), núm. 1, pp. 63-67; y “César y Cortés frente al enemigo", en Actas del Primer Congreso Internacional sobre Hernán Cortés, Universidad, Salamanca, 1986, pp. 17-26.

${ }^{18} \mathrm{Si}$ el Romancero se transmitía de forma oral, algunas crónicas fueron publicadas al poco tiempo de la introducción de la imprenta en España, como la Crónica del Cid Ruy Díaz, Tres Compañeros Alemanes, Sevilla, 1498. El estudio más completo sobre la figura histórica del Cid, en el que se recogen tanto aportaciones críticas modernas como las de las antiguas crónicas, es el de Menéndez Pidal, La España del Cid (utilizo la ed. abreviada y rev. de Espasa Calpe, Madrid, 1967); Menéndez Pidal se refirió además a la presencia del Cid en el Romancero en su Romancero hispánico, (Espasa Calpe, Madrid, 1968, t. 1, pp. 222-229); otros trabajos más recientes de interés sobre el tema son el de Jules Horrent, Historia y poesía en torno al "Cantar del Cid", Ariel, Barcelona, 1973 o las actas del congreso internacional, El Cid: de la materia épica a las crónicas caballerescas, ed. M. Alvar, Universidad, Alcalá, 2002. 


\section{El héroe militar}

Uno de los aspectos más destacados de la personalidad de Cortés, tal como éste se nos muestra en las Cartas de relación, es su extraordinario valor en la batalla: el conquistador toma desde el principio decisiones arriesgadas ${ }^{19}$; una vez en el combate, encabeza siempre a sus hombres en las situaciones de mayor riesgo ${ }^{20}$ y no cuida de sí mismo sino de ellos, quienes, al igual que los soldados de César, lo necesitan como líder y llegan a dar su vida por él ${ }^{21}$. Pero además, si, como decía Salustio, "el nervio de la guerra es el ingenio"22, Cortés va a mostrar a lo largo de todas las Cartas una extraordinaria habilidad estratégica ${ }^{23}$ que se inicia con el conocimiento de "la discordia y desconformidad" de los pueblos sometidos a Moctezuma, sobre la cual explica "no hobe poco placer, porque me paresció hacer mucho a mi propósito y que podría tener manera de más aína sojuzgarlos" (pp. 187-188).

${ }^{19}$ Como cuando manda cortar las manos de los cincuenta enviados tlaxcaltecas antes del enfrentamiento con Xicoténcatl (véase p. 179) o cuando, tras la supuesta conspiración de los cholultecas, amenaza con entrar en la tierra de Moctezuma "haciéndole todo el daño que pudiese como enemigo" sin saber todavía con qué tipo de ejército puede encontrarse (véase p. 196).

${ }^{20}$ Como ocurre en el intento de refrenar la rebelión azteca: “....yo comencé a subir por la escalera de la dicha torre y tras mí ciertos españoles... Y arriba peleamos con ellos tanto que les fue forzado saltar della abajo" (p. 275).

${ }^{21}$ Así, a propósito del cerco a la capital azteca, Cortés explica: “...como yo estaba muy metido en socorrer a los que se ahogaban, no miraba ni me acordaba del daño que podía rescebir, y ya me venían a asir ciertos indios de los enemigos, y me llevaran si no fuera por un capitán de cincuenta hombres que yo traía siempre conmigo y por un mancebo de su compañía, el cual después de Dios me dio la vida, y por dármela como valiente hombre perdió allí la suya”, y añade: “...aquel capitán que estaba conmigo, que se dice Antonio de Quiñones, díjome: «Vamos de aquí y salvemos vuestra persona, pues sabéis que sin ella ninguno de nosotros puede escapar»" (p. 396). Sobre Julio César explicaba Plutarco que "la devoción y la buena disposición de los soldados hacia él era tanta, que incluso quienes no se habían distinguido en nada de los demás en las restantes campañas se comportaban como invencibles e irresistibles a cualquier peligro en defensa de la gloria de César" (Vidas paralelas, ed. E. Crespo, Cátedra, Madrid, 2005, p. 191); la manera en la que los soldados romanos cobraban ánimos ante la presencia de César puede verse asimismo en los Comentarios (cf., como ejemplo, Comentarios de la guerra de las Galias, ed. A. Cuatrecases, Espasa-Calpe, Madrid, 2001, p. 125). Sería excesiva en este caso una comparación con el Cid, cuya sola presencia causaba el terror de sus enemigos (véase Menéndez Pidal, La España del Cid, pp. 500-501).

${ }^{22}$ Cayo Salustio, La conjuración de Catilina. La guerra de Jugurta, Espasa-Calpe, Madrid, 1986, p. 15. También decía a este respecto MAQuiavelo que el príncipe "no debe tener otro objetivo ni otro pensamiento, ni ha de tomar cosa alguna bajo su control que no sea la guerra, sus tácticas y sus reglas" (El Príncipe, p. 116).

${ }^{23}$ Sobre dicha habilidad estratégica de Cortés, pero también sobre la de Moctezuma, puede verse el interesante artículo del comandante JuAN BATISTA GonzÁLEz, "El juego de la estrategia en la conquista de México", en Actas del Primer Congreso..., pp. $67-87$. 
Dice Maquiavelo que "es necesaria la colaboración de sus habitantes a la hora de conquistar un territorio" ${ }^{24}$, y añade:

...quien se encuentre en un territorio de lengua y costumbres diferentes debe... acaudillar y defender a los menos fuertes, ingeniárselas para debilitar a los poderosos y guardarse de que, por circunstancia alguna entre allí un señor de poderío similar (p. 74).

Cortés va a seguir estas máximas al convertir en aliados a esos pueblos enfrentados al gran poder azteca, debilitando dicho poder con la prisión de Moctezuma en su propio palacio y eliminando la posibilidad de que otros españoles le arrebaten su conquista al someter al enviado de Velázquez, Pánfilo de Narváez.

Sin embargo, la rebelión azteca acaba con todos estos logros, de forma que la capacidad estratégica del conquistador español se va a poner en juego de forma esencial durante el asedio a la ciudad de Tenochtitlán. Cortés, que había intentado atraer el territorio por medios diplomáticos, asume ahora el enfrentamiento directo y definitivo. Para realizarlo, afianza su política de alianzas reforzando las existentes (con Cempoala, Tlaxcala y Cholula) y ampliándolas hacia otros pueblos ${ }^{25}$ : lo que en un principio había sido una forma de presión indirecta, de subversión, se convierte en una "coalición amplificable" ${ }^{26}$, de manera que el asedio a la ciudad de MéxicoTenochtitlán sólo se emprende cuando el conquistador español logra acrecentar suficientemente el ejército indígena aliado acogiendo a los pueblos que le llegan para rendirle vasallaje $\mathrm{e}^{27}$.

Pero además, al igual que César, Cortés utiliza su inteligencia y sus conocimientos técnicos para superar los medios del enemigo en la batalla gracias a artilugios como los tres "ingenios de madera" que Pedro Mártir identificará con las "tortugas" $\operatorname{romanas}^{28} \mathrm{y}$, sobre todo,

${ }^{24}$ El Principe, p. 71.

${ }^{25}$ Lo que le obliga a su vez a asistirlos en su defensa incluso en momentos especialmente difíciles para los españoles (cf. Cartas, pp. 335 y 400-401).

${ }^{26}$ Véase BATISTA, art. cit., pp. 80-81.

${ }^{27}$ Intensificando así una política habitual del Imperio romano, y concretamente de Julio César, como se muestra en el siguiente pasaje de los Comentarios: "Mientras César permanecía en aquel lugar para poner a punto las naves, llegaron legados de una gran parte de los morinos para excusarse por las decisiones tomadas... [y asegurarle] que ellos se comprometían a cumplir lo que les ordenase. Considerando César lo oportunamente que había llegado esa visita, pues no deseaba dejar un enemigo a su espalda... los aceptó como aliados" (op. cit., p. 167).

${ }^{28}$ Sobre estos "ingenios", explica Cortés: "cada uno llevaba veinte hombres, los cuales iban dentro porque con las piedras que nos tiraban de las azoteas no los pudiesen ofender, porque iban los ingenios cubiertos de tablas y los que iban dentro eran ballesteros y escopeteros y los demás llevaban picos y azadones y barras de hierro para horadarles las casas y derrocar las albaradas que tenían fechas en las calles” (p. 272). Cf. Pedro Mártir de Anglería, Décadas del Nuevo Mundo, est. E. O’Gorman, Porrúa, México, 1964, V Década, Libro 5, t. 2, p. 493. 
a la construcción de los trece bergantines que él mismo valora como "la llave de toda la guerra" (p. 372), ya que le van a permitir el asedio a través de la laguna (recordemos cómo César había construido también naves de guerra en el Loira ${ }^{29}$ ).

Un último aspecto que da la calidad del héroe como guerrero es la forma en que se crece en las dificultades: decía Menéndez Pidal a propósito del Cid que "lo que da carácter épico a las empresas grandiosas no es el éxito", que el héroe "debe ser medido únicamente por el valor energético de su esfuerzo" 30 : si el arrojo de Cortés se muestra de forma muy clara en la narración del asedio a México-Tenochtitlán, también es manifiesto con anterioridad, en la narración de la derrota ante los rebeldes aztecas y la retirada de la Noche triste ${ }^{31}$, e incluso en la Quinta relación, nuevo testimonio de su capacidad estratégica, ya que narra cómo se ve obligado a emplear el ingenio en su lucha contra una naturaleza hostil y destructora. En este contexto, el narrador describe como verdaderas hazañas hechos como la construcción, en el pueblo de Çaguatezpan, de un puente cuya descripción evoca la del levantado sobre el Rin por Julio César: “...tomé por postrer remedio determinarme de hacer una puente... era tal la obra que comenzamos que a todos pareció cosa imposible... lleva más de mill vigas que la menor es casi tan gorda como un cuerpo de un hombre y de nueve y de diez brazas de largo" (pp. 555-556) ${ }^{32}$.

\section{El perfecto gobernante}

Sin llegar a detenerme como debiera en este aspecto fundamental de su carácter, creo necesario recordar que, como gobernante, Cortés muestra en primer lugar su conocimiento y respeto de la ley. El legalismo es un aspecto esencial de la actuación del conquistador (incluso antes del problema jurídico que supone la fundación de Veracruz) ${ }^{33}$ que lo asemeja en cierto modo al Cid, cuya habilidad en materias jurídicas había quedado atestiguada en las crónicas y en el Romancero $^{34}$.

${ }^{29}$ Véase CÉSAR, op. cit., p. 136.

${ }^{30}$ Menéndez Pidal, La España del Cid, pp. 515-516.

${ }^{31}$ Cf., entre otros pasajes, Cartas, pp. 278, 283 y 290.

${ }^{32}$ Sobre el puente de César sobre el Rin, que le permite ser el primer hombre en atravesar este río con un ejército, cf. CÉSAR, op. cit., pp. 163-164 y Plutarco, op. cit., p. 200, donde se califica la tarea de "espectáculo superior a todo lo que se pueda creer".

${ }^{33} \mathrm{Cf}$. a este respecto, entre otros, los trabajos de VAlero Silva, op. cit. y Víctor FrankL, "Hernán Cortés y la tradición de las Siete Partidas. (Un comentario jurídico histórico a la llamada «Primera carta de relación» de Hernán Cortés)", Revista de Historia de América, 53/54 (1962), 9-74.

${ }^{34}$ En poemas como "Tres cortes armara el rey" (véase Menéndez Pidal, $L a$ España del Cid, pp. 498-500 y Romancero hispánico, pp. 222-225). 
El conquistador se muestra asimismo con una clara visión política que le va a permitir reconstruir la ciudad de México y emprender el proyecto político y económico de la Nueva España ${ }^{35}$. Pero dicho proyecto no habría sido posible sin el apoyo de los indígenas sojuzgados, a los cuales Cortés parece aplicar la máxima maquiavélica: “...quien, contra la voluntad popular, llegue a príncipe... deberá, antes que nada, ganarse al pueblo; tarea fácil siempre y cuando asuma su protección"36; este papel de protector de los indios fue un especial compromiso del conquistador, quien se cuidó de consignarlo con frecuencia en sus textos, sobre todo en la Cuarta y la Quinta relaciones ${ }^{37}$.

\section{El vasallo del rey}

Un tercer rasgo esencial del héroe cortesiano es el vasallaje a la Corona española. Como ha señalado Víctor Frankl, dicho vasallaje define ya el "retrato" de Cortés que se ofrece en la Carta de Veracruz, donde se muestra la idea de que "el valor de un hombre radica en la intensidad de su servicio al Rey en cuanto representante de Estado y Nación y en la correspondiente renuncia a su provecho personal" 38 .

La propia fundación de Veracruz se presenta como un acto de vasallaje, y la confrontación que se realiza en la correspondiente carta entre Cortés y Velázquez, se basa fundamentalmente en un problema de fidelidad al rey por parte del vasallo, que se va a intensificar en la Segunda relación durante todo el pasaje referente al enfrentamiento con Pánfilo de Narváez y sus hombres ${ }^{39}$.

Pero Cortés va a enriquecer además este concepto de vasallaje con un importante matiz: la idea de que el servicio está por encima de la posible gratitud de quien lo recibe; así, la Quinta relación finaliza con las siguientes palabras:

...yo aunque Vuestra Majestad más me mande desfavorecer no tengo de dejar de servir, que no es posible que por tiempo Vuestra Majestad no conosca mis servicios. Y ya que esto no sea, yo me satisfago con hacer lo que debo y con saber que a todo el mundo tengo satisfecho y les son notorios mis servicios y lealtad con que los fago. Y no quiero otro mayorazgo para mis hijos sino éste (p. 661).

\footnotetext{
${ }^{35}$ Véase Bartolomé Bennassar, Hernán Cortés. El conquistador de lo imposible, Temas de Hoy, Madrid, 2002, pp. 121-126.

${ }^{36}$ Maquiavelo, op. cit., p. 101.

${ }^{37}$ Cf., a modo de ejemplo, Cartas, pp. 468, 472-473, 500-501 o 636-637.

${ }^{38}$ FrANkL, art. cit., p. 42.

${ }^{39}$ Véase Cartas, pp. 248-265.
} 
Como vemos, Cortés logra una perfecta caracterización de sí mismo como vasallo del rey en este pasaje último que, como ha señalado Ángel Delgado, con una clara finalidad política (recordemos que acaba de iniciarse el juicio de residencia), evoca como ningún otro la figura literaria de ese otro vasallo ejemplar, el Cid, también desfavorecido durante cierto tiempo por su monarca. Como plantea Delgado,

La historia de el Cid es básicamente la de una incomprensión del rey Alfonso, que por malos consejos exilia a Rodrigo Díaz de Vivar, a pesar de lo cual éste se mantiene fiel a su rey y emprende conquistas en su nombre. Sus servicios militares son tan notorios que al fin el monarca debe reconocer su error, premiándole con mercedes y honores. Es evidente que Cortés evoca astutamente la figura del héroe medieval para establecer un paralelo con su propio destino ${ }^{40}$.

Cortés, sin embargo, nunca llegará a recuperar el total reconocimiento de Carlos V, y por ello esta imagen del vasallo ejemplar continuará ocupando buena parte de sus escritos posteriores, en especial en los últimos años ${ }^{41}$.

\section{El elegido de Dios}

El paralelismo casi explícito con el héroe castellano de la cita anterior nos lleva a considerar a su vez un último aspecto, también propiamente medieval, en la caracterización que Cortés ofrece de sí mismo: a lo largo de las Cartas (y éste va a ser un punto de coincidencia interesante con otros autores de la Crónica, desde el mismo Cristóbal Colón) va a elaborar de forma cada vez más destacada su imagen de elegido de Dios, de instrumento de la Providencia.

El providencialismo aparece de forma reiterada en las Cartas, como era lógico, referido a los logros de la conquista ${ }^{42}$, pero a partir de la Segunda relación observamos un proceso de "personalización" del favor divino, que se vincula en principio a las propias

${ }^{40}$ Nota de Delgado a su edición de las Cartas, p. 661.

${ }^{41}$ Cf. Documentos cortesianos, t. 4, pp. 234-235. La última carta que dirige al monarca, fechada en Valladolid a 3 de febrero de 1544, recoge, a través de la amarga queja al monarca, el sentido último de este vasallaje que CoRTÉs ha ido mostrando a lo largo de sus textos: “...ha cuarenta años que me he ocupado en no dormir, mal comer ya las veces ni bien ni mal, traer las armas a cuestas, poner la persona en peligros, gastar mi hacienda y edad, todo en servicio de Dios, trayendo ovejas a su corral muy remotas de nuestro hemisferio, e ignotas y no escritas en nuestras Escrituras, y acrecentando y dilatando el nombre y patrimonio de mi rey" (Documentos cortesianos, t. 4, p. 267).

${ }^{42}$ Cf. como ejemplos, Carta de Veracruz, p. 131; Segunda relación, pp. 177-178, 275; o Tercera relación, pp. 411, 414, 427. 
decisiones ("paresció que Nuestro Señor me inspiró..." ${ }^{43}$ ) y que ya en la Cuarta relación va a implicar la definición de uno mismo como instrumento divino: "Dios Nuestro Señor fue servido de me hacer medio por donde veniese en su conoscimiento y debajo del imperial yugo de Vuestra Alteza..." (p. 522).

Con esa imagen religiosa, Cortés completa las distintas vertientes de una configuración literaria del héroe a la que contribuyen a su vez determinados rasgos de su escritura que se aprecian especialmente en su caracterización desde el punto de vista militar y político, como son, entre otros, el hábil manejo del sujeto gramatical, el "tono mesurado, ecuánime, impasible del relato"44 (uno de los motivos más claros para su comparación con Julio César ${ }^{45}$ ) o la elipsis (la cuidadosa omisión de determinadas informaciones que reducirían su protagonismo, comprometerían su imagen de leal vasallo o dejarían al descubierto los errores cometidos) ${ }^{46}$. Protagonista sólo de grandes acciones, perfecto en sus cualidades morales, militares y políticas, pero sobrio al referirse a sí mismo, Cortés se inscribe en la Historia como héroe capaz de conquistar una gran nación para su rey y de gobernarla con justicia.

\section{A MODO DE CONCLUSIÓN: ¿EL TRIUNFO DEL HÉROE?}

Como ya explicó Marcel Bataillon, la prohibición en 1527 de las tres cartas cortesianas publicadas hasta entonces (la segunda, tercera y cuarta), testimonia, más allá de los pretextos aducidos para la mis$\mathrm{ma}^{47}$, el temor de un rey que, lejos de confiar en su vasallo, ve el peligro de que "el conquistador de la Nueva España se convierta en héroe fundador y epónimo del nuevo «reino»"48, un temor al que habían

${ }^{43}$ Cf., como ejemplos, pp. 180, 327 y 626-627.

${ }^{44}$ Ramón Iglesia, Cronistas e historiadores de la conquista de México. El ciclo de Hernán Cortés, El Colegio de México, México, 1980, p. 17.

${ }^{45}$ Decía Menéndez Pelayo que “...hay pocas escrituras, en ninguna lengua, que por la noble sencillez, por la modesta grandeza, por la serena objetividad, por la plácida lisura con la que se refieren las cosas más extraordinarias, recuerde o emule tanto los Comentarios de César como estas Relaciones del conquistador de la Nueva España, con ser documentos oficiales, escritos al compás de los acontecimientos, y sin ningún propósito literario" (en Flasche, art. cit., p. 149).

${ }^{46}$ Cito solamente estos rasgos que serán objeto detenido de un futuro trabajo.

${ }^{47}$ El principal de ellos es la queja que recibe la Corona por parte de Pánfilo de Narváez, quien dice haberse visto agraviado en los escritos cortesianos. La carta de Carlos V a Narváez al respecto comienza: "Bien sabéis cómo a vuestro pedimento y suplicación, nos, mandamos dar una nuestra cédula, por la cual mandamos que ningún librero ni impresor ni otra persona, imprimiese ni tuviese ciertas cartas y relaciones que Hernando Cortés nos había enviado de las cosas acaescidas en la Nueva España..." (Documentos cortesianos, t. 1, p. 465).

${ }^{48}$ Marcel Bataillon, "Hernán Cortés, autor prohibido", en Libro jubilar de Alfonso Reyes, Porrúa, México, 1963, p. 80. 
contribuido esas otras vertientes, las del guerrero y gobernante, ofrecidas por el conquistador hasta la Cuarta relación, en la que Cortés "se erguía claramente en actitud de legislador y hombre providencial que sabría gobernar al país tan bien como supo conquistarlo" 49 .

Es por ello que, de algún modo, la prohibición de las Cartas testimonia también el triunfo de Cortés en esa configuración de su imagen heroica, una imagen que pronto sería trasmitida a su vez por los cronistas coetáneos, a los que el conquistador logró fascinar con su escritura. Los elogios a Cortés llegaron de la mano de biógrafos entusiastas, como López de Gómara o el anónimo autor del texto latino $D e$ rebus gestis Ferdinandi Cortesii, pero también de los que en un principio fueron más recelosos con el autor, como Pedro Mártir de Anglería o Gonzalo Fernández de Oviedo. Fue precisamente este último quien, hacia 1545, escribió en el Libro XXXIII de su Historia general y natural de las Indias:

...es muy justa cosa que en la memoria de los que viven, estén escriptas las hazañas e fechos memorables de Hernando Cortés... porque son cosas raras e peregrinas e no tienen semejanza ni comparación con ejército ni cerco alguno de aquellos que por muy famosos [historiadores] están escriptos de los pasados ${ }^{50}$.

Paradójicamente, quien por aquel entonces comenzaba a convertirse en el héroe literario de la crónica, el romance o la poesía épica, era un hombre "viejo y pobre", cercano ya a una anónima muerte en su casa sevillana, relegado por un Imperio que extendía con mano firme su dominio en el continente americano.

BeAtriz Aracil Varón

Universidad de Alicante

${ }^{49} I d$. Éste es asimismo el motivo, para este autor, de la posterior prohibición del libro de Gómara, concebido como biografía del conquistador; sobre otros aspectos que pudieron influir en la retirada de la crónica de Gómara, cf. Iglesia, op. cit., pp. 118-129.

${ }^{50}$ Gonzalo Fernández de Oviedo, Historia general y natural de las Indias, ed. J. Pérez de Tudela, BAE, Madrid, 1959, t. 4, p. 142. El autor no llegó a publicar esta parte de su magna obra. 
